\title{
PUESTA EN MARCHA DEL ÁREA DE TERAPIA OCUPACIONAL EN SERVICIO DE REHABILITACIÓN, EN EL HOSPITAL EL CARMEN
}

\author{
STARTING THE AREA OF OCCUPATIONAL THERAPY IN REHABILITATION SERVICES \\ "HOSPITAL EL CARMEN"
}

\section{Paulina Alegría V. ${ }^{1}$ y Evelyn Puga P. ${ }^{2}$}

\begin{abstract}
Resumen
Los cambios demográficos y epidemiológicos asociados con el envejecimiento poblacional e incremento de enfermedades crónicas producen un aumento de las demandas en salud. Por este motivo, en los próximos años el Estado contempla la apertura de servicios de medicina física y rehabilitación que incorporen el área de terapia ocupacional en la red pública asistencial.

El objetivo del presente documento es transmitir la experiencia de la puesta en marcha del área de terapia ocupacional en un hospital público. Se identifican 10 etapas a lo largo del proceso de implementación y puesta en marcha, cada una de ellas con sus respectivos facilitadores y obstaculizadores. Los principales resultados son la confección de protocolos de atención, de documentos técnicos asociados a la intervención, el fortalecimiento de la coordinación con la red de discapacidad, la terapia ocupacional como disciplina necesaria en estas unidades, el aumento de la demanda de atención, la buena recepción de los usuarios y la importancia de los equipos de trabajo.

Como conclusiones destacan las habilidades particulares del equipo de trabajo para el desarrollo de procesos, la coordinación con la red para clarificar la pertinencia de ingreso de usuarios y los procesos de referencia y contra referencia. Y por último, la importancia de sistematizar la experiencia que facilite el traspaso de información a equipos que puedan ejecutarlo posteriormente.
\end{abstract}

\section{Palabras clave:}

puesta en marcha, medicina física y rehabilitación, terapia ocupacional, hospital público.

\begin{abstract}
Demographic and epidemiological changes associated with aging population and increasing chronic diseases result in increased health demands. For this reason the State plans to open services physical medicine and rehabilitation incorporating the area of occupational therapy in the coming years.

The objective of this document is to convey the experience of the implementation of the area of occupational therapy in a public hospital. Ten stages are identified throughout the implementation process and implementation, each with their facilitators and obstructionist. The main results are translated into the making of care protocols, technical documents associated with the intervention, strengthening coordination with the network of disability, occupational therapy as necessary discipline in these units, increased demand for care, the good reception of users, the importance of the work teams, and systematization document.

As findings highlight the particular skills of the work team development process, coordination with network to clarify the relevance of user input, reference and counter processes. And finally, the importance of systematizing the experience that facilitates the transfer of information to teams that can run later
\end{abstract}

\section{Keywords:}

experience, commissioning, physical medicine and rehabilitation, occupational therapy, public hospital.

Fecha de recepción: 19/07/2016.

Fecha de aceptación: 21/10/2016.

1 Terapeuta ocupacional, licenciada en ciencia de la ocupación. Terapeuta ocupacional en Hospital Clínico Metropolitano La Florida. Email: paulina.alegria@gmail.com.

2 Terapeuta ocupacional, licenciada en ciencia de la ocupación. Candidata a magíster en salud pública. Email:eve.puga.p@gmail.com. 


\section{INTRODUCCIÓN}

Con las aperturas y proyectos de nuevos hospitales en Chile, los cuales cuentan en su totalidad con servicios de medicina física y rehabilitación, surge un nuevo desafío para la terapia ocupacional (TO) (División de gestión de redes asistenciales, 2015): planificar, implementar y ejecutar esta especialidad como parte del equipo rehabilitador en salud pública a nivel nacional. Si bien es cierto que hace años trabajan terapeutas ocupacionales dentro de los hospitales, el trabajo en unidades centradas en la rehabilitación que cuenten con un equipo completo constituido por kinesiólogos, fonoaudiólogos, terapeutas ocupacionales, psicólogos, trabajadores sociales y fisiatras es reciente en el sistema público, más aún en regiones (Vergara, 2010).

En octubre de 2013, El Hospital el Carmen de Maipú inicia esta "vivencia" con la conformación del staff de terapia ocupacional. Para este proceso se buscan experiencias similares que sirvan de guía, sin embargo no se encuentran sistematizaciones o referencias que contribuyan a orientar y anticipar las problemáticas que implica iniciar proyectos de tal envergadura.

Es así como surge la necesidad de plasmar las acciones que permitieron llevar a cabo la puesta en marcha del servicio de TO del Hospital El Carmen de Maipú, con los aciertos, errores, cambios, dificultades propias del sistema de salud nacional y el desconocimiento del quehacer profesional de TO, para que así puedan ser de utilidad para quien lo necesite y logren facilitar o al menos aportar en los aspectos que deben considerarse al momento de habilitar unidades similares.

El siguiente documento describe la experiencia de dos terapeutas ocupacionales que dieron apertura al área de terapia ocupacional del servicio de medicina física y rehabilitación del Hospital El Carmen de Maipú Este trabajo busca transmitir la vivencia y reflexión a los terapeutas ocupacionales que lo requieran, basándonos en el aumento de la oferta laboral asociada a la construcción de nuevos hospitales públicos

A continuación -en el marco teórico- se abordará un poco de historia de los servicios de medicina física y rehabilitación en Chile, la probable demanda asistencial y finalmente el aporte e importancia de la terapia ocupacional en el equipo rehabilitador.

\section{MARCo teÓrico}

\subsection{Un poco de historia: Desarrollo de la medicina física y rehabilitación en Chile}

Los servicios de rehabilitación, según lo convenido por el comité de expertos de la Organización Mundial de la Salud (OMS) en rehabilitación médica en 1968, tienen por función la "aplicación coordinada de un conjunto de medidas médicas, sociales, educativas y profesionales para preparar o readaptar al individuo con objeto de que alcance la mayor proporción posible de capacidad funcional" (OMS, Segundo informe del comité de expertos de la OMS de rehabilitación médica, 1969). En Chile, estos servicios se comienzan a conformar en el año 1958, con el Dr. Livio Paolinelli Monti, quien estudia la especialidad de fisiatría en Estados Unidos en la Universidad de Colorado, Denver. En 1961 se inaugura el servicio de medicina física y rehabilitación (SMFR) en el Hospital Clínico de la Universidad de Chile y en 1964, cuando se inicia la formación de residentes, da el impulso a esta especialidad médica, contando al 2010 con 23 médicos residentes (Vergara, 2010).

Los servicios de salud públicos incluyen la intervención de profesionales de la rehabilitación como kinesiólogos, terapeutas ocupacionales y/o fonoaudiólogos, pero no todos poseen servicios de medicina física y rehabilitación constituidos como tal es, que además de estos profesionales contemplen la atención del fisiatra como médico de cabecera. Además existen estos servicios en instituciones de salud privada como el Hospital Clínico de la Universidad de Chile, Clínica Las Condes y Clínica Alemana, entre otras, junto a la atención en clínicas o instituciones especializadas en esta área como la clínica Los Coihues y los institutos de rehabilitación infantil, aquellas derivadas de servicios de salud asociados a las Fuerzas Armadas como DIPRECA y CAPREDENA y finalmente la atención en rehabilitación brindada en mutualidades como la Asociación Chilena de Seguridad (ACHS), la Mutual de Seguridad y el Instituto de Seguridad del Trabajo (IST).

Actualmente, el Gobierno de Chile contempla en su plan de inversiones en infraestructura de salud 60 nuevos hospitales en 4 años (MINSAL, plan de inversiones de infraestructura en salud, 2014), lo que significa mayores oportunidades de apertura de servicios de me- 
dicina física y rehabilitación y por consecuencia una mayor oferta de empleos para los profesionales de la rehabilitación.

\subsection{Escenario actual: Demanda para los servicios de medicina física y rehabilitación}

La demanda asistencial para los servicios de medicina física y rehabilitación (SMFR) se focaliza en personas que presentan patologías que involucran diversas afecciones como aquellas del sistema nervioso, del sistema musculo-esquelético enfermedades degenerativas, entre otras, las cuales requieren tratamientos especializados que permitan mejorar su salud y fomentar su reinserción social. Dicha demanda, según lo señalado por el comité de expertos de la OMS en rehabilitación médica, se gesta desde periodos tempranos en el tratamiento (OMS, Segundo informe del comité de expertos de la OMS de rehabilitación médica, 1969), es decir, desde que la persona se encuentra hospitalizada por un episodio agudo o desde las primeras consultas médicas que confirmen el diagnóstico.
La demanda a los servicios de medicina física y rehabilitación que podría provenir de la hospitalización se puede inferir desde los datos del Ministerio de Salud respecto a los egresos hospitalarios. Según estos, se evidencia que de todos los grupos de enfermedades según CIE-10, las enfermedades del sistema nervioso, de la piel y tejido subcutáneo, del sistema circulatorio, del sistema osteomuscular, traumatismos y malformaciones congénitas concentran el $25 \%$ de los egresos hospitalarios, es decir, 279.778 egresos considerando un total anual de 1.623.875, situación que se repite todos los años desde 2008. Si bien el porcentaje es solo la cuarta parte de todos los egresos hospitalarios, dichas enfermedades generan un impacto en la salud pública asociado a que en su mayoría superan el promedio de días de estada en la hospitalización (MINSAL, Egresos hospitalarios, 2012), lo que provocaría un gasto público elevado y justificaría la necesidad de un tratamiento de rehabilitación precoz. La siguiente tabla muestra los promedios de días de estada por grupo de enfermedad:

Tabla No1:

Promedio estadía que podrían demandar al SMFR.

\begin{tabular}{|c|c|}
\hline & 5,7 \\
\hline Enfermedades del sistema nervioso central (G00-G99) & 7,9 \\
\hline Enfermedades de la piel y del tejido subcutáneo (L00-L99) & 6,6 \\
\hline Enfermedades del sistema osteomuscular y del tejido conjuntivo (M00-M99) & 3,9 \\
\hline Malformaciones congénitas, deformidades y anomalías cromosómicas (Q00-Q99) & 6,7 \\
\hline Traumatismos, envenenamientos y algunas otras consecuencias de causas externas (S00-T98) & 5,9 \\
\hline Enfermedades del sistema circulatorio (I00-I99) & 8,0 \\
\hline
\end{tabular}

Fuente: Elaboración propia a partir de datos del MINSAL sobre "egresos hospitalarios" (MINSAL, Egresos hospitalarios, 2012).

Otra fuente de la que se puede inferir la demanda corresponde a los estudios que caracterizan la situación de la discapacidad en Chile. El estudio realizado en 2004 nos muestra que el 12,9\% de la población nacional presenta algún tipo de discapacidad, es decir 2.068.072 personas, de los cuales el 31,3\% corresponde a personas con discapacidad de causa motora, un $27,7 \%$ de causa sensorial $(8,7 \%$ auditivo y $19,0 \%$ visual), un $7,8 \%$ de causa mental, intelectual $9 \%$, viscerales $13,9 \%$ y un $10,3 \%$ múltiple. Considerando estos datos se puede inferir que aquellos con discapacidad motora y múltiple son usuarios que podrían requerir directamente los servicios de medicina física y rehabilitación, los cuales corresponden al 41,6\% (INE, 2004), es decir, a 860.318 personas aproximadamente. Por otra parte, el estudio reciente de 2015, referido a la población mayor de 18 años, señala que en este grupo existe un $20 \%$ de personas en situación de discapacidad, esto es, 2.606.914 personas, lo que nos refleja- a pesar de la acotación en la edad, a diferencia del estudio anterior-- 
un gran porcentaje de personas que requerirían algún apoyo terapéutico (Chile, 2016).

Las fuentes anteriormente mencionadas proveen información relevante respecto a la posible demanda de los SMFR; sin embargo, junto con ellas, se debe considerar el nuevo escenario que trae consigo la transición demográfica que posiciona al envejecimiento poblacional como un fenómeno sin precedentes. Datos de la OMS estiman que entre 2000 y 2050 se duplicará la población mundial mayor de 60 y más años, pasando del $11 \%$ al $22 \%$, siendo la de mayor crecimiento el grupo de personas de 80 años y más (OMS, Envejecimiento y ciclo de vida). Dentro de este contexto, Chile no es la excepción, ya que según la CEPAL, el país se encuentra en etapa de transición avanzada, puesto que muestra bajos niveles de mortalidad y fecundidad, junto con una tasa de crecimiento del orden del $1 \%$, generando un aumento sostenido de la población envejecida (CEPAL, 2007). Dicha situación implica reconocer que las enfermedades crónicas tendrán cada vez mayor presencia en la morbilidad de la población, determinando la necesidad en aumento de atención especializada para aquellos casos donde las patologías afecten la autonomía e independencia de las personas (OMS, Envejecimiento y ciclo de vida). Por lo tanto, se puede inferir que habría un aumento sostenido en la demanda de los SMFR.

Frente a estas exigencias resulta preponderante el establecimiento de servicios de medicina física y rehabilitación para la atención hospitalaria y ambulatoria, con el fin de desarrollar dispositivos especializados que permitan prevenir la dependencia y mejorar la calidad de vida de las personas.

\subsection{Terapia ocupacional: Disciplina esencial en los servicios de medicina física y rehabilitación}

El desarrollo de la terapia ocupacional (T.O.) en medicina física comienza de forma incipiente en la primera guerra mundial y fuertemente en la segunda, relacionado con el gran número de personas sobrevivientes que quedaban "lisiadas" (Gómez, S. y Ruedas, L., 2002). Por su parte, en Chile se hizo necesaria la incorporación de estos profesionales tras la creación del Servicio Nacional de Salud en 1952, la consolidación de las mutuales en 1958, los cambios demográficos como la disminución de la mortalidad y el aumento de la tasa de nacidos vivos como consecuencia de las mejoras en el sistema de sa- lud, que produjo un aumento de sobrevida de personas con secuelas físicas evidentes, la epidemia de la poliomelitis en la década de los 50 y 60 y el aumento de niños con secuela de parálisis cerebral. Dichos hitos constituyen la base para el progreso de la profesión y para la visibilización de la disciplina como esencial en los equipos de salud (Gómez, S. y Ruedas L., 2002).

Respecto a la importancia de la T.O. en los servicios de medicina física y rehabilitación, Silvia Gómez sostiene que el objetivo de la intervención es "impedir la dependencia, potenciar un rendimiento ocupacional exitoso y favorecer la inserción social" (Gomez, S., 1994). Por otra parte, Begoña Polonio hace referencia a que el objetivo de la T.O. es "intervenir en casos de déficit en el desarrollo del individuo, aplicando la actividad como medio preventivo o terapéutico, con la finalidad de lograr la armonización del sujeto en relación con su ocupación, trabajo y ocio, buscando la integración social" (Polonio, B., 2004). De forma concreta, un estudio chileno reciente con alta validez estadística evalúa el impacto del tratamiento de terapia ocupacional en adultos mayores con delirium que se encuentran hospitalizados, el cual concluye que la intervención precoz e intensiva de T.O. previene el delirium, reduce los días de estadía y mejora la independencia de estos usuarios (Álvarez, E. et al, 2012). Es por tanto fundamental la incorporación de la disciplina dentro de esta área, con el fin de ser parte de un equipo que potencie la autonomía a través de una intervención integral y de calidad.

\section{Objetivos}

El objetivo central es "Transmitir la experiencia de la apertura del área de terapia ocupacional del servicio de medicina física y rehabilitación del Hospital El Carmen de Maipú".

Los objetivos específicos corresponden a:

- Explicar la experiencia de la apertura del área de terapia ocupacional del servicio de medicina física y rehabilitación del Hospital El Carmen de Maipú en etapas.

- Reflexionar respecto a los principales resultados de proceso.

- Proponer una discusión respecto a la temática. 


\section{MéTOdo}

\subsection{Metodología}

La metodología que se utilizará será la descripción de una experiencia a través de los siguientes pasos:

A) Revisión del estado del arte de la temática: origen de los SMFR en Chile, posible demanda asistencial e importancia de la terapia ocupacional en el área.
B) Recolección de datos a partir de la experiencia de 2 terapeutas ocupacionales que iniciaron el proyecto del SMFR del Hospital El Carmen de Maipú. Aquí se conformaron mesas de trabajo donde se analizó la experiencia de los años 2013 y 2014.

C) Reflexión y discusión de la recolección de los datos.

\subsection{Descripción de la experiencia}

Para describir la experiencia se establecieron una serie de etapas, las cuales se configuran en un orden temporal que permitirá visualizar los hitos más importantes del proceso. Al comenzar se presenta una figura con las etapas identificadas.

Figura $\mathrm{N}^{\circ} 1$ :

Etapas de apertura de SMFR: Experiencia del área de T.O.
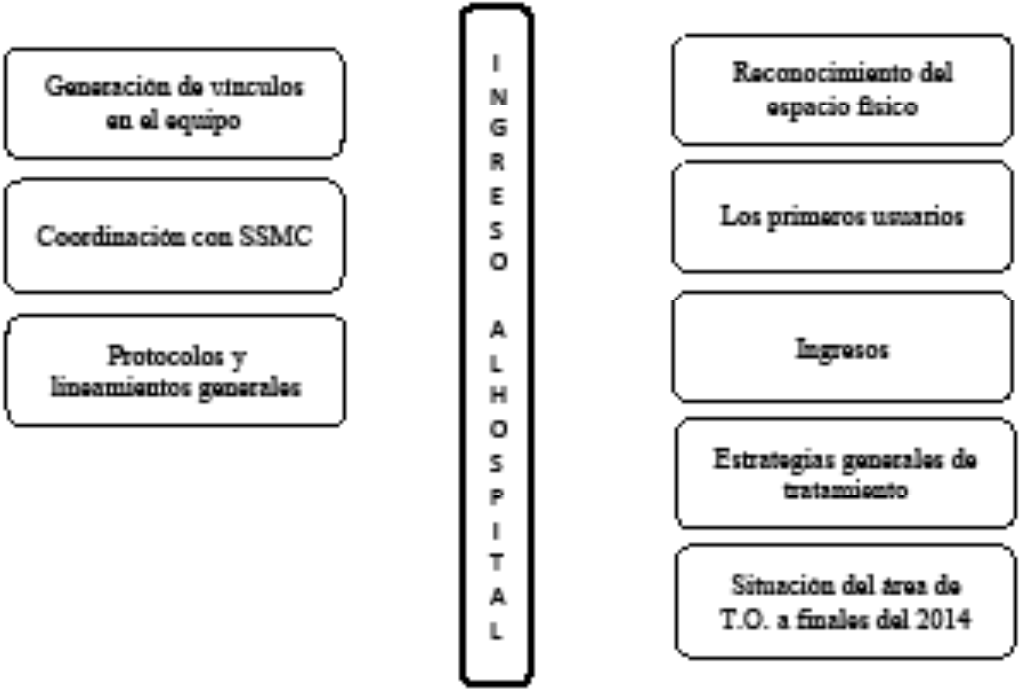

Fuente: Elaboración propia.

\subsubsection{Generando vínculos en el equipo de rehabilitación}

A mediados de octubre de 2013 se integran dos de los tres cargos de terapia ocupacional (TO) al equipo de medicina física y rehabilitación del Hospital El Carmen de Maipú (HEC), que para esa fecha ya cuenta con un fisiatra como jefe de unidad y cinco kinesiólogos. Debido a este desfase en los tiempos de ingreso, es el equipo de kinesiología quien acoge y orienta en los primeros momentos a los terapeutas ocupacionales (TTOO), facilitando información y material de apoyo que guíe los primeros pasos en el incipiente desarrollo del área. 
En esta primera etapa se establecen los lazos que se hicieron vitales e indispensables para la posterior habilitación de las unidades en terreno, el trabajo en conjunto y los esfuerzos necesarios para levantar un proyecto de semejante envergadura.

Antes de finalizar esta etapa ya se integra la tercera colega restante, junto con el comienzo de las capacitaciones del sistema computacional Florence, el cual organiza la atención clínica de los usuarios.

\subsubsection{Coordinación con la red de rehabilitación en el Servicio Metropolitano Central (SSMC)}

El HEC pertenece al SSMC, red que previamente se constituía por el Hospital de Urgencia de Asistencia Pública (HUAP), Hospital San Borja Arriarán (HSBA) y los centros de atención de salud de las comunas de Santiago Centro, Maipú, Cerrillos y Estación Central. Para comenzar la integración a esta red, se coordina una reunión con la mayoría de dichos establecimientos, el objetivo de presentar el servicio de medicina física y rehabilitación, mencionar las prestaciones que se planean realizar, el perfil de usuarios a recibir y contrastarlo con las necesidades, condiciones de trabajo y criterios de atención de los demás organismos de la red.

Una vez ahí, se establece un vínculo directo con las TTOO del HSBA, aspecto fundamental para la posterior gestión de visitas y orientación para el levantamiento del área cuando se logre habilitar el espacio del HEC.

\subsubsection{Protocolos y lineamientos generales}

Teniendo la noción del trabajo de colegas en la red, los lineamientos de atención que requieren los usuarios de Maipú y Cerrillos, y con la experiencia de cada una de las TTOO, se inicia la confección de los protocolos de atención de niños, adultos y adultos mayores. Así, se establece la cartera de prestaciones disponibles, los criterios de inclusión y exclusión -dados principalmente por zona de residencia u hospital de referencia-, tiempos de atención de controles y consultas (ingresos) (30 a 60 minutos, respectivamente), número de sesiones estimadas, y en base a ello, los requerimientos de materiales a gestionar, pautas de evaluación a aplicar y algunos planes de actividades a desarrollar. Este material se escribe y se envía para revisión y autorización de las jefaturas correspondientes.

\subsubsection{Reconocimiento del espacio físico}

Durante el mes de noviembre se visita y conoce el espacio de medicina física y rehabilitación, ubicado en el primer piso del hospital, el cual consta de tres salas destinadas a la atención de TO. Los planos presentados establecen una sala para órtesis, otra de actividades de la vida diaria (AVD) y una tercera para atención general. Sumado a ello, existe un pequeño gimnasio para pediatría destinado a consulta de kinesiología y/o TO.

Todas las salas cuentan con pequeñas ventanas que permiten el ingreso de luz natural y leve recambio de aire, aspecto fundamental debido a la falta de aire acondicionado en las salas de atención de usuarios. Sin embargo, no se cumplen los requerimientos para las órtesis de PVC en relación a espacios, ventilación y equipamiento de las profesionales.

A medida que se atiende a los usuarios, los espacios dentro de la unidad empiezan a verse limitados, principalmente para la atención de niños y para el tratamiento de lesiones de mano por parte del equipo de kinesiología. Es así como se debe buscar apoyo de neurólogos para gestionar un espacio de atención de pediatría fuera del servicio, espacio que logra gestionarse y habilitarse en el segundo piso, sector de consultas de neurología.

Una vez despejados los espacios, los cuales al llegar estaban siendo utilizados como bodega de otros materiales, se realizan labores de aseo, se solicita mobiliario y se habilitan para la atención de público.

\subsubsection{Primeros Caracterización de los usuarios}

Los primeros usuarios que ingresan a TO son aqueIlos que se encuentran en lista de espera para atención de la especialidad de fisiatría. Luego de la evaluación con el especialista se realiza la derivación a TO para evaluación. En su mayoría, los usuarios son adultos y adultos mayores con enfermedades crónicas y de larga data. Las patologías más comunes presentadas por los usuarios fueron: accidente cerebrovascular con más de un año de evolución (secuelados), artritis reumatoide, artrosis y amputaciones vasculares. 


\subsubsection{Ingresos}

Los ingresos de TO se retrasaron en comparación con el estamento de kinesiología, puesto que no llegaban materiales para el trabajo. Debido a la latencia en la respuesta de los administrativos en relación a los insumos y materiales, se decide comenzar a implementar la sala de TO con herramientas, juegos y actividades de las propias profesionales, entre los cuales se encontraban: juegos de estimulación cognitiva, juegos de mesa, argollas, tablero de Montessori y ganchos de ropa, entre otros.

Paralelamente, se comienza la utilización del sistema computacional Florence, el cual registra ingresos como "consulta" y sesiones como "controles". Se registran las primeras consultas y se realizan las evaluaciones de los primeros usuarios a través del formato de evaluación confeccionado en la protocolización.

\subsubsection{Estrategias iniciales de tratamiento}

Luego de las primeras evaluaciones se identifican características comunes en los usuarios como el diagnóstico de enfermedad crónica y de larga data, la mayoría con cuidador y adultos mayores. Por esta razón, y junto con la escasez de materiales, se decide realizar talleres como estrategia inicial y como forma de liberar la lista de espera. Del análisis se generaron 3 talleres que corresponden a:

- Taller para usuarios independientes "Soy SeguriTO": taller para usuarios independientes en su desempeño ocupacional, pero con patologías que les producían dolor o tenían alta probabilidad de caídas.

- Taller de promoción de vida independiente: taller para usuarios que tenían un cuidador, pero que presentaban alto potencial de mejorar su desempeño ocupacional.

- Taller para el cuidador "Yo también importo": taIler para cuidadores de usuarios totalmente dependientes.

Para los tres talleres se gestionan los horarios y el lugar de realización. Se llevan a cabo los dos primeros talleres, puesto que el último no tenía demanda de usuarios. Cada taller consta de 4 sesiones que incorporaban trípticos informativos. Los talleres incluían las siguientes temáticas: impacto del dolor y de la movilidad restringida, rutinas saludables, redes de apoyo, técnicas de protección articular, técnicas de conservación de la energía, higiene postural, manejo ambiental, ayudas técnicas, técnicas de autocuidado, prevención de caídas, técnicas de manejo del dolor, resolución de problemas (autonomía versus independencia), entrenamiento en actividades de la vida diaria y estimulación cognitiva.

De acuerdo a la experiencia de las profesionales hubo falencias que no permitieron continuar con esta metodología, las cuales correspondieron a inasistencias reiteradas, baja convocatoria y tope con las sesiones de kinesiología. Por lo tanto, se requirió un cambio en la estrategia focalizado en la replicación de estas mismas sesiones pero de forma individual.

El aspecto positivo fue la creación de material educativo útil para las sesiones, que se sigue usando en la actualidad.

\subsubsection{Ambulatorio y hospitalizados}

Posterior a los talleres, las modalidades de atención de TO eran en policlínico (ambulatoria) y en sala (hospitalizados). En un principio, las 3 terapeutas compartían los roles, pero debido a la alta carga asistencial de los usuarios ambulatorios se tomó la decisión de que una terapeuta realizara media jornada solo en hospitalizados. Desde ese momento se generó el equipo de "hospitalizados" contemplando las áreas de kinesiología, terapia ocupacional, fonoaudiología y fisiatría.

Paralelamente comienzan las reuniones clínicas de todo el servicio, donde se discuten aspectos administrativos y se realizan presentaciones de temáticas por estamento. Junto con esto, se inicia la participación de TTOO en la red de discapacidad municipal como representantes del servicio.

Además comienzan a ser derivados niños y niñas desde el servicio de neurología infantil, por lo que se destinan estas prestaciones a dos terapeutas ocupacionales.

El esquema final de atención es el siguiente:

Tabla No 2 :

Primera distribución de atención de T.O.

\begin{tabular}{|c|c|c|c|}
\hline & \multirow{2}{*}{ Hospitalizados } & \multicolumn{2}{|c|}{ Ambulatorio } \\
\cline { 3 - 4 } & & Niños & Adultos \\
\hline T.O. 1 & $\mathrm{X}$ & & $\mathrm{X}$ \\
\hline T.O. 2 & & $\mathrm{X}$ & $\mathrm{X}$ \\
\hline T.O. 3 & & $\mathrm{X}$ & $\mathrm{X}$ \\
\hline
\end{tabular}

Fuente: Elaboración propia. 


\subsubsection{Cambios en el perfil del usuario}

A causa de estas reuniones de coordinación se comienza a definir un nuevo perfil de usuarios asociados a la pertinencia diagnóstica y al nivel de atención. Los criterios corresponden a: usuarios con problemas neurológicos de menos de un año de evolución, con artritis y artrosis severas (leves y moderadas corresponden a atención primaria), niños (con problemas del desarroIlo, incluyendo trastornos motores, cognitivos y generalizados del desarrollo), cicatrices de menos de 6 meses de evolución y amputados vasculares (se mantiene).

Los usuarios que eran derivados por los fisiatras y que no correspondían a este nivel de atención eran atendidos en 2 o 4 sesiones, para posteriormente ser derivados a su sala de rehabilitación en la atención primaria de salud.

\subsubsection{Situación del área de T.O. a finales de 2014}

Se continúa la redistribución de funciones debido a que frente a la gran demanda se contrata a otra terapeuta ocupacional solo para atención de niños y niñas con trastornos generalizados del desarrollo derivados de neurología infantil; mientras que para el servicio de medicina física y rehabilitación se destinan solo aquellos niños con trastorno motor y menores de 2 años.

Junto con esto, se destinan horas de terapeuta ocupacional para atención específica de niños hospitalizados, en UPC infantil y neonatología, por lo tanto la distribución de atención actual se encuentra en la siguiente tabla:

Tabla No3:

Segunda distribución de atención de T.O.

\begin{tabular}{|c|c|c|c|c|}
\hline & \multicolumn{2}{|c|}{ Hospitalizados } & \multicolumn{2}{c|}{ Ambulatorio } \\
\hline & Niños & Adultos & Niños & Adultos \\
\hline T.O. 1 & & $\mathrm{X}$ & & $\mathrm{X}$ \\
\hline T.O. 2 & $\mathrm{X}$ & & $\mathrm{X}$ & $\mathrm{X}$ \\
\hline T.O. 3 & & & $\mathrm{X}$ & $\mathrm{X}$ \\
\hline T.O. 4* & & & $\mathrm{X}$ & \\
\hline
\end{tabular}

Fuente: Elaboración propia.

*TO dependiente del área de neurología infantil.
Debido a la demanda se generan nuevas prestaciones asociadas la indicación de ayudas técnicas y con la inserción laboral, por lo que se realizan los protocolos correspondientes.

En este periodo ocurre un cambio de administración en el hospital debido al cambio de gobierno, lo que provoca retrasos en la llegada de recursos materiales, situación que se perpetúa hasta el día de hoy.

\section{ANÁLISIS E INTERPRETACIÓN DE LA}

\section{EXPERIENCIA}

Para realizar un análisis de la experiencia desde nuestra interpretación, se plantean una serie de preguntas que permiten la reflexión y otorgan una visión general del impacto de la vivencia. Las preguntas son desarrolladas a continuación:

\section{1. ¿Qué cambios se han producido a nivel local con la experiencia?}

La apertura de un nuevo hospital genera expectativas en toda la población, en relación al impacto sanitario vinculado con el acceso a una atención de salud más oportuna y adecuada a los problemas de salud del territorio. En el caso del Hospital El Carmen de Maipú, estas expectativas estaban sobredimensionadas porque incorporan una promesa política cumplida y la apertura de servicios que estaban, hasta ese entonces, lejanos debido a la referencia de los usuarios al Hospital San Borja Arriarán, lo que determinaba largos trayectos y también mucha espera.

EI SMFR en particular responde a una necesidad del SSMC, el cual no poseía la atención de médico fisiatra en ninguno de sus hospitales, lo que retrasaba la atención de las personas que presentaban problemas de salud que desencadenaban una situación de discapacidad. Con el establecimiento del servicio se lograría la atención con esta especialidad y por consecuencia reducir la lista de espera.

Con dichas expectativas, se constituye la apertura del SMFR, siendo éste junto a otras modalidades ambulatorias las primeras en funcionamiento dentro del hospital, estableciéndose como el conejillo de indias que permitiría visualizar las primeras impresiones de los usuarios. El establecerse como uno de los primeros 
servicios en abrir determinó un arduo trabajo anterior y desafíos hacia el futuro. Respecto al trabajo previo, según la experiencia se puede señalar que fue necesario realizar análisis de la literatura, la creación de protocolos de atención de kinesiología y terapia ocupacional y vínculos con la red para la derivación pertinente de los usuarios.

En relación a los desafíos se consideran: la apertura de una nueva red de atención de salud para personas en situación de discapacidad, que debe brindar atención especializada y en periodo agudo o crítico, la disminución de la lista de espera de especialidad (Fisiatría) y constituirse como un servicio de atención oportuna y bien evaluado por nuestros usuarios.

En la actualidad, como se reconoce en la salud pública, las múltiples necesidades de las personas generan mayores demandas que en su momento no se visibilizaban por la ausencia de la oferta pública, como reevaluaciones, controles periódicos por fisiatras y recaídas de los usuarios. Al día de hoy el área de terapia ocupacional no mantiene lista de espera, lo cual se ha resuelto con lógica sanitaria asociada a la organización del número de sesiones pertinentes por severidad de la discapacidad y buena derivación a la red.

\section{2. ¿Qué factores influyeron positivamente? ¿y negativamente? ¿Se superaron? ¿De qué manera?}

\subsubsection{Facilitadores}

Dentro de los aspectos que favorecieron el proceso se encuentra: la motivación en el emprendimiento de un nuevo proyecto, el fuerte vínculo de amistad entre los colegas, la coordinación y colaboración y las habilidades personales de cada integrante, asociados con la proactividad y la iniciativa.

En particular, en el grupo de terapeutas ocupacionales se generó un vínculo de colaboración que fomentó un buen trabajo de equipo, logrando desarrollar diversas tareas complementarias como:

- Elaboración de protocolos de atención adulto y pediátrico.

- Distribución eficiente de los roles (agenda, estadística y adquisiciones, entre otros).

- Participación en red de discapacidad.

- Distribución de roles en atención clínica (atención cerrada y ambulatoria, adulta y pediátrica).
- Visualización de la terapia ocupacional como profesión esencial en el servicio de medicina física y rehabilitación y en diversas áreas tanto adulta o pediátrica, hospitalizada y ambulatoria.

En el desarrollo de las tareas, las terapeutas ocupacionales presentan adecuadas habilidades relacionadas con la creatividad e iniciativa, puesto que a pesar de las condiciones adversas del entorno -como la falta de materiales y espacios- logran eliminar las barreras mediante la creación y confección de herramientas de trabajo con materiales personales, la utilización de insumos destinados a otras áreas, como el yeso para realizar órtesis y uso de tubigrip, entre otros.

Otro factor facilitador del proceso es el compromiso de los propios usuarios, quienes frente a la falta de recursos proporcionan los propios para el desarrollo de los procesos de intervención.

\subsubsection{Obstaculizadores}

Los principales obstaculizadores para el equipo fueron la falta de recursos materiales junto con espacios físicos reducidos, los cuales no interfirieron en el óptimo funcionamiento de los procesos de intervención para los usuarios, que veían en la atención una oportunidad de escucha y solución de sus problemas; junto con esto, el esmero de los propios profesionales que cubrían la falta de recursos con estrategias alternativas o complementarias que satisfacían sus procesos. Estas dificultades estaban asociadas con los cambios constantes en la gestión hospitalaria, como con cambios de director, de profesionales y administrativos.

Por otra parte, se evidencia falta de liderazgo en la jefatura del SMFR que provocaba retraso en las peticiones y falta de apoyo en el requerimiento de recursos físicos y humanos.

En particular, las terapeutas ocupacionales se han enfrentado al desconocimiento de la disciplina por parte de algunos profesionales del hospital, lo que ha determinado un esfuerzo constante por posicionarse en áreas desconocidas y mostrar su trabajo y los resultados de éste. El visibilizar una disciplina genera aumento de la demanda de atención, por lo que a pesar de mantener una lista de espera de atención ambulatoria cubierta, no se puede decir lo mismo en hospitalización donde no se logra brindar un servicio continuo, por lo que existe la necesidad de contar con este recurso humano. 


\subsubsection{Soluciones}

La principal fortaleza del SMFR es su equipo profesional que ha enfrentado los problemas de manera creativa y ha dado solución a la atención de los usuarios a pesar de las limitaciones de recursos humanos y materiales. Esto ha permitido realizar un seguimiento a las demandas de recursos materiales a través de la distribución de roles dentro del equipo.

Además se gestó el cambio de la jefatura del SMFR debido a un requerimiento del equipo y de la propia jefatura anterior, siendo retomada por un fisiatra que ha dado respuestas principalmente en la coordinación, pero no ha logrado revertir el problema de recursos.

La demanda de atención de terapia ocupacional está contenida en la atención ambulatoria, mientras que en atención cerrada u hospitalización solo se realiza la evaluación y se atienden casos urgentes de personas con alto potencial de rehabilitación.

\section{2. ¿Cuáles son los principales resultados de la experiencia?}

Esta experiencia permite visualizar las exigencias, facilitadores y obstaculizadores del proceso de apertura de un servicio de atención de salud de baja complejidad como es medicina física y rehabilitación, en particular el área de terapia ocupacional. Los principales resultados de la experiencia fueron:

- Experiencia para todo el equipo en la habilitación y puesta en marcha de un servicio, considerando labores de coordinación, inventario, distribución de roles, ordenamiento de agenda, estadísticas, protocolización y ejecución de labores propias del puesto de trabajo.

- Confección de protocolos de atención del área de terapia ocupacional para adultos y niños, lo que permite tener una guía de trabajo que sustente las estrategias y de respuesta a los procesos de acreditación en salud.

- Confección de diversos documentos técnicos para la intervención de terapia ocupacional, para el tratamiento de personas con secuelas leves, moderadas y severas, protocolo de ayudas técnicas, de inserción laboral, personas quemadas y atención de pediatría y de neonatología, entre otros complementarios con kinesiología.
- Fortalecimiento del vínculo de coordinación con red de discapacidad y red de salud, especialmente con centros de rehabilitación comunitaria, sala de rehabilitación municipal y Hospital San Borja Arriarán.

- Visualización de la terapia ocupacional como disciplina necesaria en la intervención de personas en situación de discapacidad.

- Aumento de la demanda de atención de terapia ocupacional, que implica la exigencia de más profesionales.

- Se logra visualizar la importancia de los equipos de trabajo en el desempeño de la intervención, basada en su compromiso, iniciativa y motivación por lograr que el proyecto avance.

\section{DISCUSIÓN}

Durante los últimos años, el aumento de la esperanza de vida a raíz de las mejoras en salud genera una relación proporcional con el envejecimiento poblacional. Las enfermedades crónicas y la discapacidad en sus diversas esferas aumentan y hacen necesario la conformación de servicios especializados en abordaje integral y rehabilitación de estos usuarios. La demanda por acceder a estos servicios de rehabilitación es creciente, siendo necesaria su disposición dentro de instituciones de salud pública. En la actualidad, el Gobierno ha comprometido la construcción y/o remodelación de más hospitales públicos que contemplan en su totalidad la presencia de estos servicios (MINSAL, 2015).

Desde lo observado, y a pesar de la innovación que el Estado realiza al conformar estos servicios, la visión biomédica de la salud y la focalización en estrategias de sobrevida de los usuarios- aún imperantes en nuestro sistema- resta importancia al desarrollo y potenciación de los servicios de rehabilitación, lo que se traduce en una limitación en la asignación de recursos humanos, financieros, de espacio, etc. Esto en desmedro de favorecer la calidad de vida del usuario y su familia, quienes posterior a una problemática de salud aguda que cursa con una hospitalización, requieren de la atención del equipo de rehabilitación, pero por los escasos recursos acceden a estas atenciones de forma tardía. Lamentablemente, las gestiones en salud actuales desarrollan inversiones focalizadas en atención secundaria 
principalmente, es decir, cuando ya se ha instalado la enfermedad; y les es difícil reconocer la relevancia de mejorar las estrategias de atención primaria (promoción y prevención) y terciaria (rehabilitación).

A medida que se inicia la puesta en marcha de los diversos servicios en el hospital, crece la demanda por atenciones de TO que no se contemplan al comienzo del proyecto, ya que los cargos gestionados se planifican para las derivaciones de fisiatría y principalmente en modalidad abierta (ambulatoria). Sin embargo, transcurridos algunos meses, y al iniciar la difusión "puerta a puerta" del rol de TO en los diversos servicios del hospital, comienzan a llegar las interconsultas provenientes de atención cerrada como unidades de pacientes críticos, unidad de cuidados medios, unidad básica, unidades de modalidad dual como pediatría, traumatología y otras de modalidad ambulatoria como neurología infantil, reumatología, e incluso desde otros organismos de la red de salud correspondiente -las cuales no llegan necesariamente por mediaciones de fisiatría- ponen en jaque al sistema, colapsando las horas de atención disponibles y dejando de manifiesto la precariedad de recursos y la lentitud del sistema de salud para responder a las demandas que surgen y la contingencia actual. Esto genera un trabajo de alta demanda para los profesionales, no solo en términos de atención clínica, sino en la gestión e implicancias administrativas que conlleva evidenciar los déficit del sistema, generar las estadísticas, solicitar materiales e insumos y buscar las estrategias que permitan ir dando la mejor atención posible a los pacientes con los pocos elementos que se tienen y en coordinación con las jefaturas respectivas.

En base a lo expuesto, cabe señalar que el perfil de TTOO que ingresen a estos puestos de trabajo deben ser profesionales comprometidos con su labor, resilientes, con alta tolerancia a la frustración, creativos y proactivos, capaces de transformar cada desafío presentado en una oportunidad para el desarrollo y crecimiento de TO en el contexto local y generar evidencias que permitan la validez de la profesión trascendiendo a implicancias nacionales. Es decir, las habilidades personales de cada profesional serán fundamentales en los lineamientos que tomará el área de TO y en las posibilidades y campos que logren abrirse en los diversos hospitales que inicien estas unidades.
EI MINSAL cuenta con documentación respecto a lineamientos de atención de los profesionales de rehabilitación, sin embargo dichas sugerencias ministeriales, en la experiencia de las profesionales, no están ampliamente difundidas y no son conocidas por las jefaturas de estos servicios, por ende la implementación real queda sujeta a los criterios de cada institución basándose en realidades locales.

A pesar de las dificultades presentadas, se destaca la participación de los usuarios, quienes comprenden y conocen las problemáticas del sistema. Esto permite un vínculo estrecho entre TO-paciente, el cual favorece el proceso de rehabilitación, la adherencia al tratamiento y el compromiso con el contexto local, estableciendo un trabajo conjunto y activo para levantar las unidades, facilitando el empoderamiento de los pacientes como sujetos de derecho, los cuales tienen la obligación de exigir atención de salud digna, oportuna y de calidad, la cual muchas veces se ve transgredida y sin posibilidades de mejora.

\section{REFERENCIAS BIBLIOGRÁFICAS}

Álvarez, E. Garrido, M., González, F., Guzmán, E., Donoso, T., Gallegos, S., Vergara, S., Aranda, R., Prieto, S., Briceño, C., Tobar, E., Alzamora, C., Bolvarán, C, Concha, C., Valencia, F. Villalobos, F. (2012). Terapia Ocupacional precoz e intensiva en la prevención del delirum en adultos mayores ingresados a unidades de paciente crítico, ensayo randomizado, resultados prelimiares. Revista Chilena de Terapia Ocupacional, Vol. 12, №1, pág. 44-59.

CEPAL (2007). La dinámica demográfica de América Latina. Proyección de la población. Observatorio demográfico №3, pág. 9-15.

División de gestión de redes asistenciales, MINSAL (2015). Puesta en marcha informe de Julio 2015. Santiago.

Gobierno de Chile (2016). Resultados del II Estudio Nacional de la Discapacidad en la población adulta. Recuperado en Junio de 2016, de http://www.gob.cl/resultados-del-ii-estudio-nacional-de-la-discapacidad-en-la-poblacion-adulta/.

Gómez, S. y Ruedas, L. (2002). Bases para el desarrollo de la Ciencia de la Ocupación. Santiago.

Gómez, S. (1994). Cuaderno de Terapia Ocupacional. Santiago.

INE (2004). Prevalencia de Personas con Discapacidad en Chile. Recuperado en Diciembre de 2015, de http://doi.org/10.4321/S1134928X2009000200007. 
MINSAL (2012). Egresos hospitalarios. Recuperado en diciembre de 2015, de http://intradeis.minsal.cl/egresoshospitalarios/menu_publica_nueva/menu_publica_nueva.html.

MINSAL (2014). Plan de inversiones de infraestructura en salud. Recuperado en diciembre de 2015, de Minsal: http://web.minsal.cl/ plan_nacional_inversiones_salud.

OMS, (1969). Segundo informe del comité de expertos de la OMS de rehabilitación médica. Recuperado de http://apps.who.int/iris/bitstream/10665/38490/1/WHO_TRS_419_spa.pdf.

OMS (s.f.). Envejecimiento y ciclo de vida. Recuperado en diciembre de 2015, de http://www.who.int/features/factfiles/ageing/ageing_ facts/es/.

Polonia, B. (2004). Terapia Ocupacional en discapacitados físicos: teoría y práctica. Madrid: Editorial Médica Panamericana S.A.

Vergara, L. (2010). Desarrollo de la Medicina Física y Rehabilitación como especialidad médica. Rev. Hosp. Clín. Univ. Chile 2010; 21: 281-8. 